ألحافظ: استخدام الشبكة العصبية الاصطناعية في التشغيل الأمثل لمحطات التوليد الكهرومائية للفترة الطويلة

$$
\begin{aligned}
& \text { استخدام الثنبكة العصبية الاصطناعية في التثغيل الأمثل لمحطات التوليد الكهرومائية } \\
& \text { للفترة الطويلة }
\end{aligned}
$$

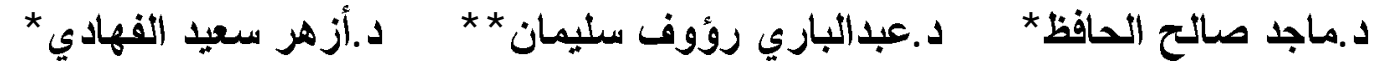

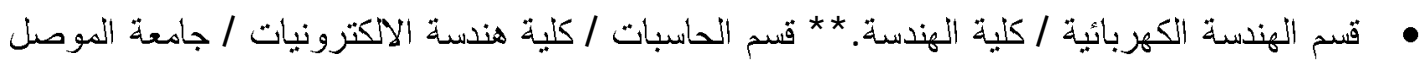

$$
\begin{aligned}
& \text { الخلاصة } \\
& \text { يعرف توزيع الحمل في منظومة تحوي محطات توليد حرارية ومحطات توليب كهرومائية بالتوزيع الأمثل للحمل، وتكون دالــة }
\end{aligned}
$$

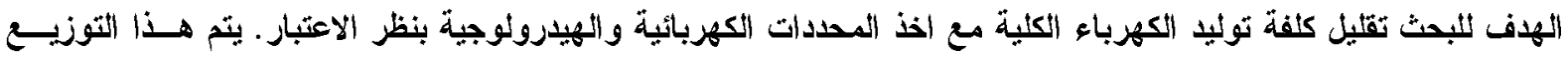

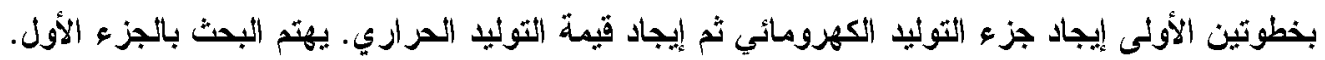

$$
\begin{aligned}
& \text { تم في هذا البحث استخدام تقنية الثبكة العصبية الاصطناعية لإيجاد التوزيع الأمثل للمياه الواردة على أثــهر البــنة. }
\end{aligned}
$$

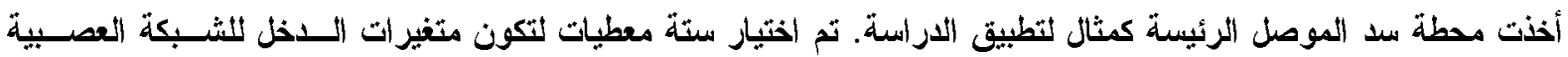

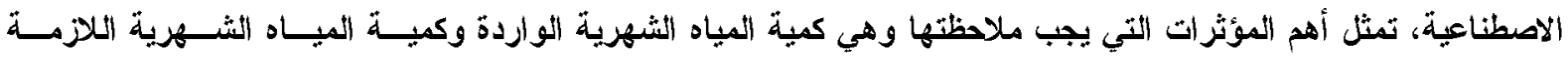

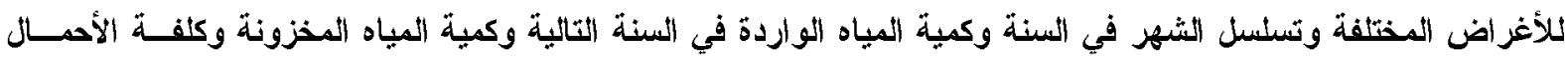

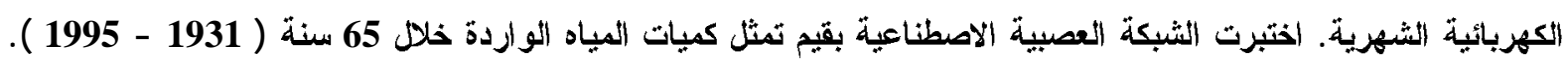

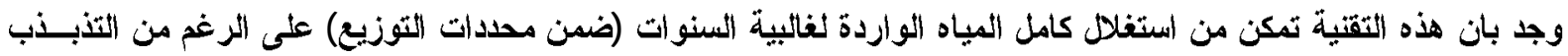

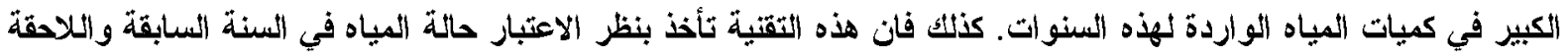

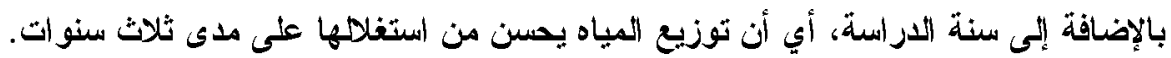

\title{
Optimal Hydrothermal dispatch for Long Term Using ANN
}

\author{
Dr. M. S. Al-Hafid Dr. A. B. R. Sulaiman Dr. A. S. Al-Fahadi
}

\begin{abstract}
:
The optimal hydrothermal scheduling is the distribution of load among the generating stations. The objective function for the problem is to minimize total generating cost considering the electrical and hydrological constraints. This problem is usually solved in two stages. The first is to find the hydropower generation share, then to find the thermal generation share. This research concerns the first stage. This work uses the Artificial Neural Network (ANN) to find the optimal scheduling of the monthly water discharge over the year. The main power station of Mosul dam is considered as an application example for this study. Six input variables are chosen to be the input to the ANN. They are monthly inflow water, monthly demand water, number of the month in a year, expected next year water inflow, available stored water (water from the past year). The ANN is trained and tested by the available water flow data over the past 65 years (1931- 1995). It is found that this technique enables the utilization of whole inflow water for most of the years (within considered constraints) in spite of the great fluctuation of inflow water for these years. Besides, this technique takes into account the status of the water for last year and next year in addition to the year under study. This means that the water distribution improves the utilization of available water over three years
\end{abstract}


تساهم السدود في زيادة كفاءة استغلال المو ارد المائية، بتتظيم المياه في نهر معين وخزن الفائض منهــا

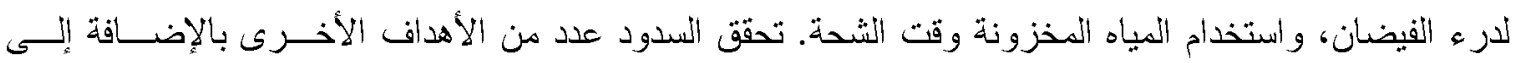

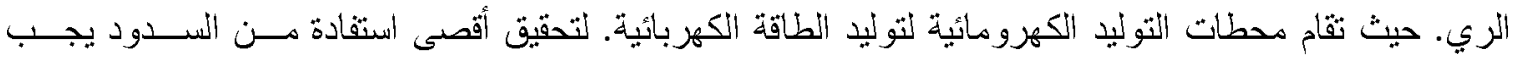
الموازنة والتنسيق بين المنطلبات والمحددات المختلفة وذلك عن طريق التحكم بتوزيع التصريف على أنثهر السنة، ومنها توليد الكهرباء. يعرف توزيع الحمل في منظومة تحوي محطات توليد حر ارية ومحطات توليد كهرومائيـة

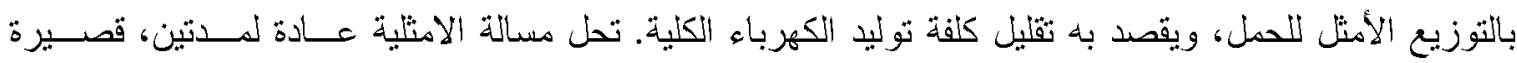

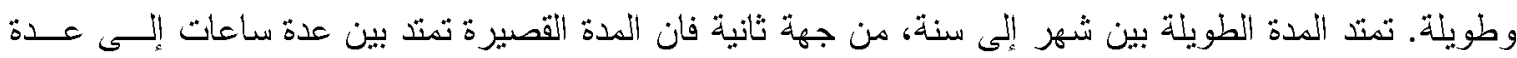

تمثل مسالة التوزيع الأمثل إحدى المسائل المهمة والمعقدة. استخدمت طرق برمجة مختلفة لحل هذه المسالة منها الطرق الخطية وغير الخطية [3,2,1]. حديثا استخدمت تقنية الخوارزمية الجينية للوصول إنى التوزيع الأمثل للمباه على أتثهر السنة [5,4 تمثل المصادر (1 -5) نماذج للبحوث المنشورة حول حل المسالة بالطرق التقليدية وتثقيــة الخوارزميـة

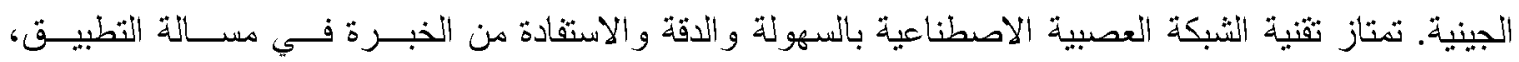

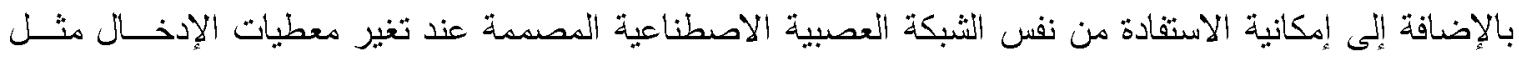

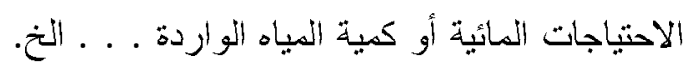

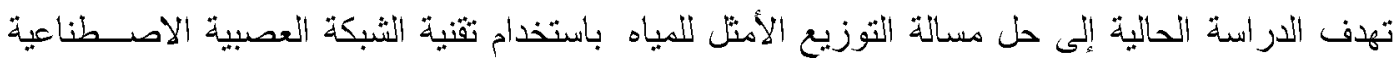

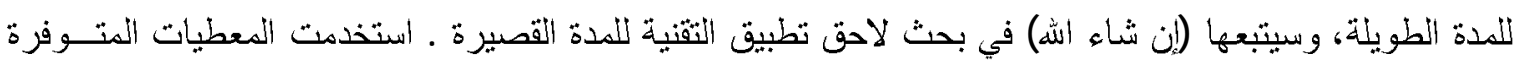

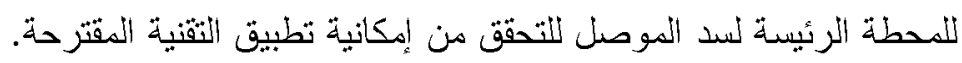

\section{2 - المتطلبات و المحددات :}

يمثل نهر دجلة وسد الموصل نموذج للسدود و الأنهار التي تحتاج إلى سيطرة مبرمجة للاستفادة مــن الميــاه لتلبية الأهداف المختلفة. تتذبذب كمية المياه التي يحملها نهر دجلة عند سد الموصل بين سنة وأخرى. يمثل الثكل رقم

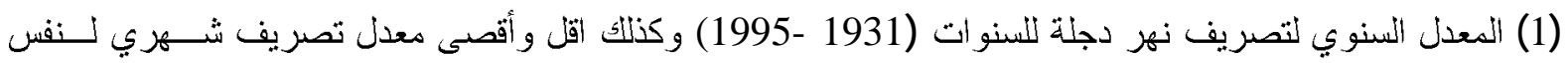

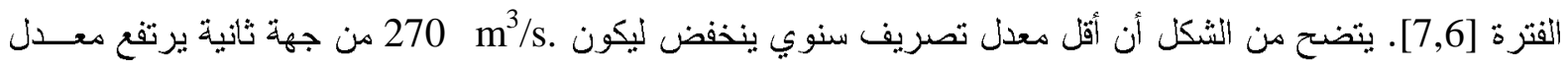

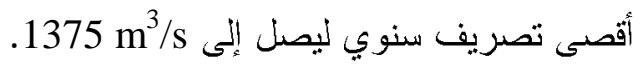

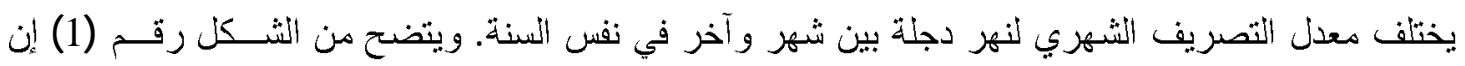
اقل معدل تصريف شهري هو 98 m³ وأقصى معدل تصريف شهري في نفس السنة هو

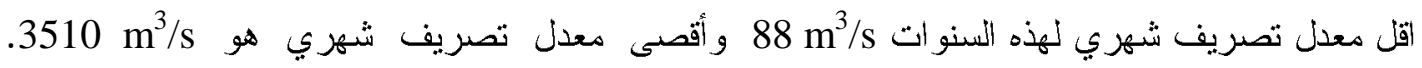

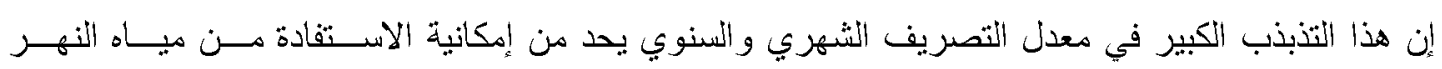
ويتطلب نتظيم إطلاق مياه السد للوصول إلى أقصى استفادة من المياه المتاحة وضمن المتطلبات المتعددة التالية: 
ألحافظ: استخدام الشبكة العصبية الاصطناعية في التشغيل الأمثل لمحطات التوليخ الكهرومائية للفترة الطويلة

أ - متطلبات الكمبات المائية:

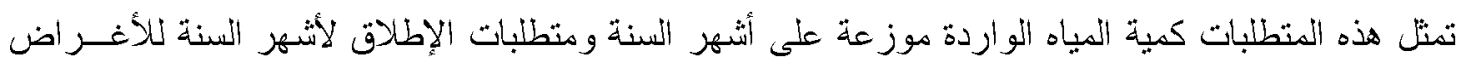

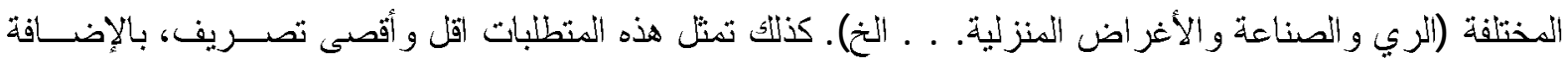

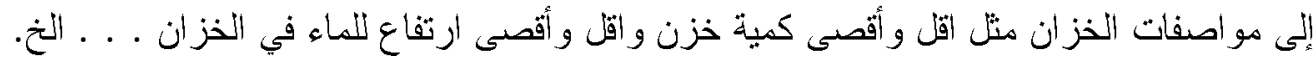

\section{المعل واعلى واقل تصريف شهري لنهر دجلة}

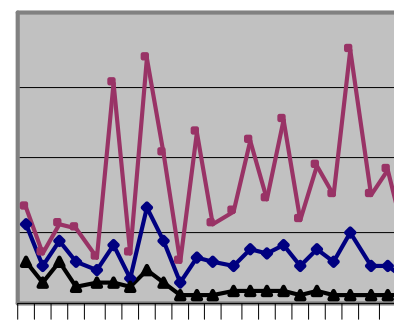

$$
\begin{aligned}
& \text { ஏ } \\
& \text { السنوات }
\end{aligned}
$$

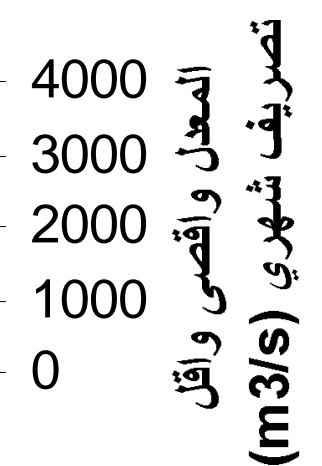

الثكل رقم 1 : المعدل واقل وأقصى تصريف شهري لنهر دجلة للسنوات 1931 -1995.

\section{ب - متطلبات محطة توليد الكهرباء و المنظومة الكهربائية:}

تمثل متطلبات محطة توليد الكهرباء اقل و أقصى كمية إطلاق للمياه وأقل و أُعلى ارتقاع للمياه في السد. وتمثل

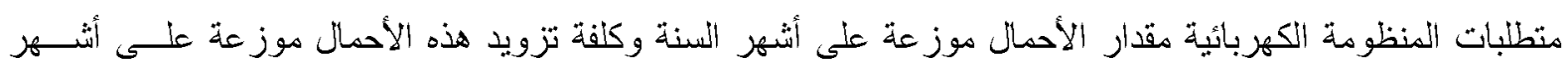
السنة كذلك.

ج - هدف الار اسـة:

يؤدي تذبذب المياه في أُثهر السنة والسنوات المتعاقبة (عند غياب تنظيم المياه المطلقة) إلى استحالة الاستفادة

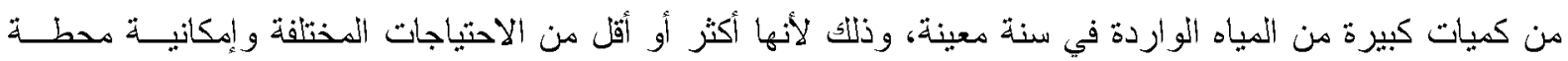

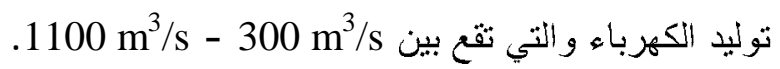
يهدف البحث الحاني إنى تتظيم المياه المطلقة من سد الموصل لتقي بالمتطلبات و المحددات المـذكورة ســابقا،

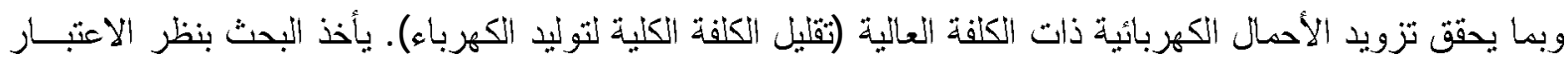

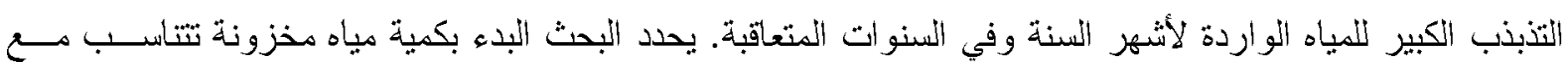

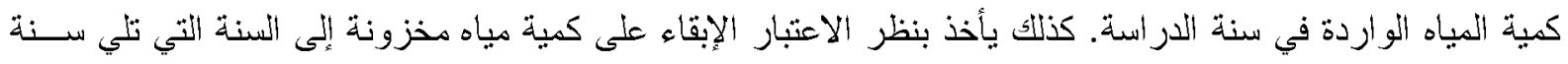

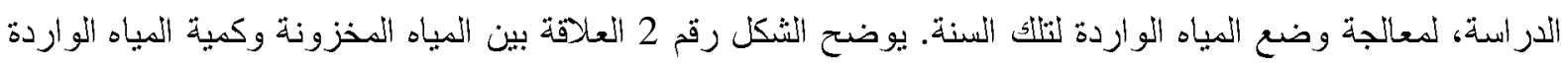

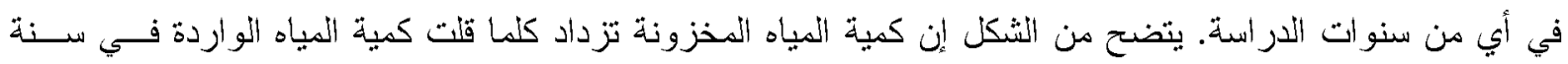


الدر أسة. أُي إن البحث يستفاد من مياه النسنة السابقة لسنة الدراسة وكذلإك يوفر كمية مياه مناسبة لحالة المياه في النسـنة التي تلي سنة الدر اسة، بالإضدافة إلى توزيع المياه لسنة الدر استة.

\section{العلاقة بين المياه الواردة سنويـا والميـاه المخزونـة}
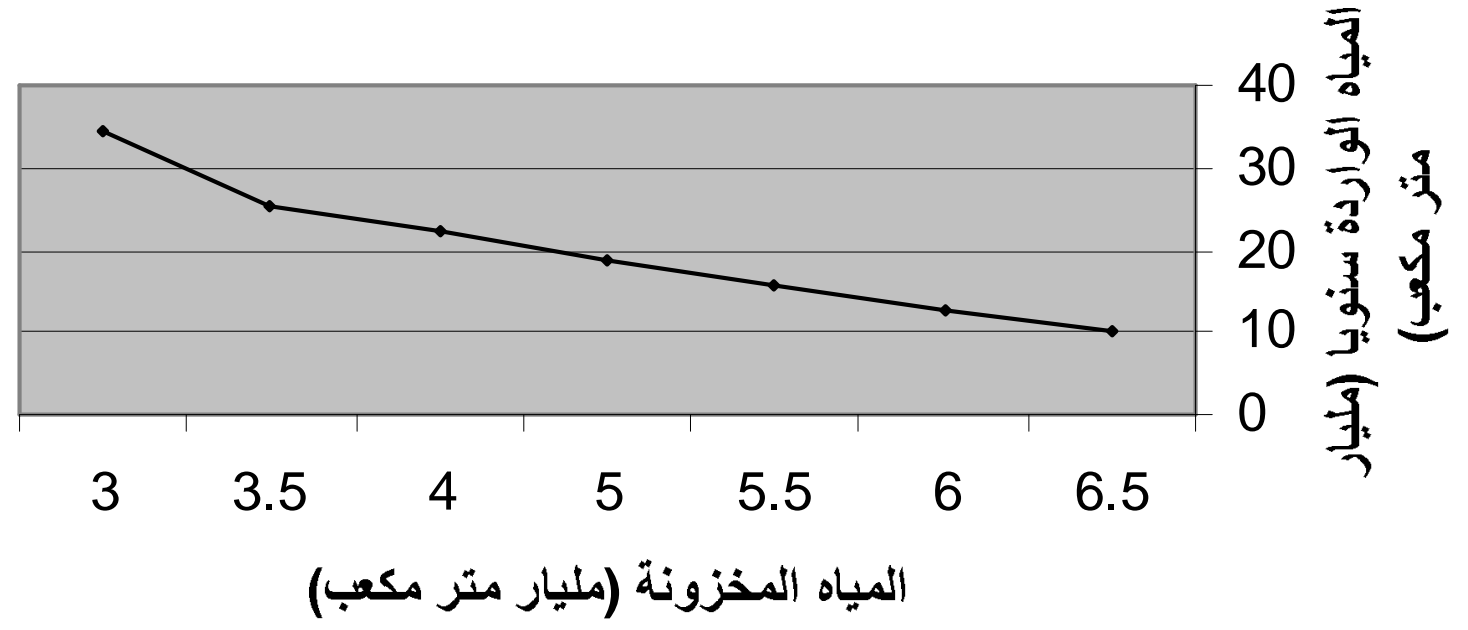

الثُكل رقم 2 : العلاقة بين المياه المخزونة والمياه السنوية الواردة.

\section{3 - الشبكة العصبية الاصطناعية :}

تعرف ألأنظة الذكية (Intelligent systems) بأنها التتي تتعلم ويتحسن أداؤها بالتدريب [8, 9, تمثل تقنية الثبكة العصبية الاصطناعية إحدى هذه الطرق الأكية. إن نظرية الشبكات العصبية الاصطناعية معروفة بشكل جبد. وهي تستخدم لحل معضلات في تخصصات مختلفة. دربت الثبكات العصبية الاصطناعية لتتجز دالات مركبة في مجالات تطبيق مختلفة وتثنمل تمبيز النماذج، وتصنيف الكلام، ومنظومات السيطرة والإشر اف. ويمكن تدريب الثبكات العصبية هذه الأيام لتحل مسائل كتلك التي يصعب حلها باستخدام طرق البرمجة التقليدية.

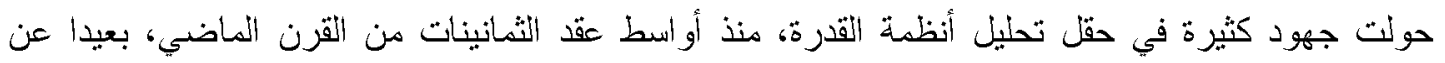
هنهجية التمثيل الرياضي التقليدي والتي تأتي من حقول بحوث العمليات ونظرية السبطرة و التحليلات العددية باتجاه التثنية الأول صر امة من الذكاء الاصطناعي (Artificial Intelligence AI). تتلخص تقنية الثنبكة العصبية الاصطناعية بثلاث خطوات، الأونى تكوين الثنبكة العصبية الاصطناعية و الثانية تدريب الثبكة العصبية الاصطناعية المكونة والثالثة اختبار الثبكة العصبية الاصطناعية. يتم عادة تقسيم المعطيات المتوفرة إنى جزثين يستخدم الأول لتدريب الثنبكة العصبية الاصطناعية و الثناني لاختبار الثبكة العصبية التبية الاصطناعية. تصبح الثبكة العصبية الاصطناعية جاهزة بعد التأكد من نتائج الاختبار . تم في هذا البحث استخدام الثبكة العصبية الاصطناعية (ANN) في الحصول على التهنية التوزيع الأمثل للمياه

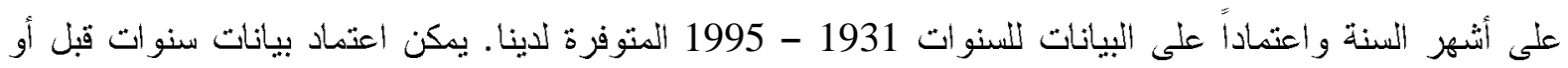


بعد هذا التاريخ عند توفر ها، كما بمكن إعادة التدريب عند حصول تغير كبير في النبيانات مثل تغير كمية المياه الواردة

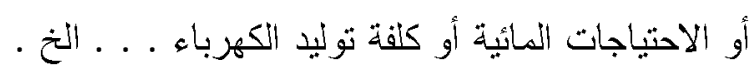

\section{3}

تم استخدام شبكة عصبية نوع ألانتشار العكسي (Back propagation) ذات طبنتين. تحتوي الطبقة الاؤلـى (Hidden layer) أن عدد الإخر اج المطلوب في النطبيق، هو الذي يحدد عدد العقد في هذه الطبقة.

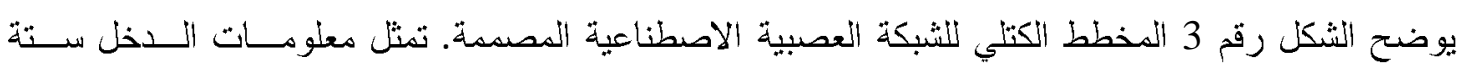
متغير ات. اختيرت هذه المتغير ات لتمثل المؤثرات المهمة التي يجب ملاحظتها عند تقريز كمية المياه المطلقة في شهر معين. وهي كالآتي:

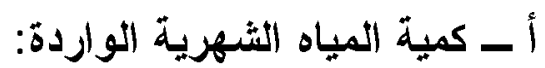

تمثل كمية المياه الواردة أهم العو امل المؤثرة. تختلف كمية المياه الواردة بين شهر و آخر ـ تكـــون الميــاه

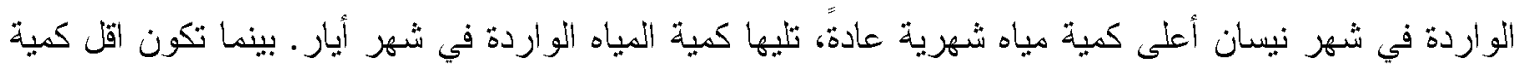

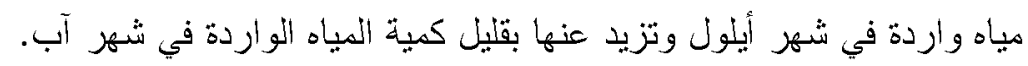

\section{ب - كمبة المباه الثهرية لنلأغر اض المختئل}

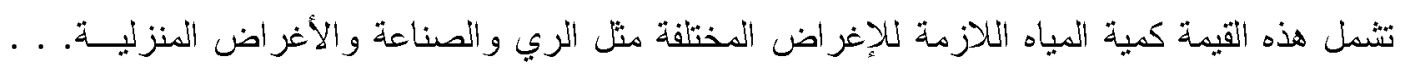
الخ. وهي من العوامل المهمة المحدد_لكمية المياه المطلقة، والتي يجب إن تلبيها. تحدد هذه الاحتياجات مسبقا ويتم الالتززام بها عند نوزيع المياه على أُشهر السنة.

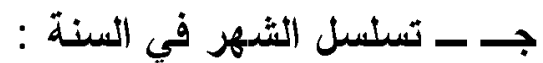

يمثل تسلسل الشهر في النسنة احد العوامل التي تساهم في زيادة التوفيق بين كمية المياه الواردة مع كميــة

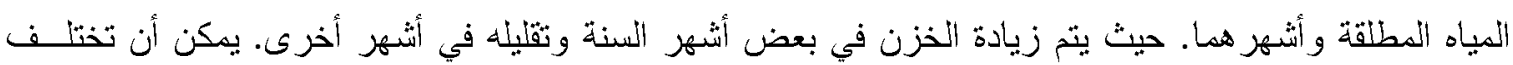

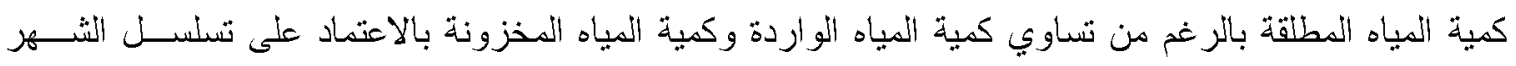

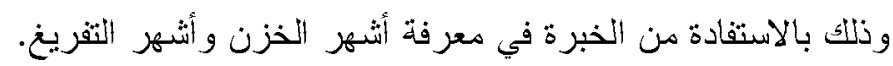

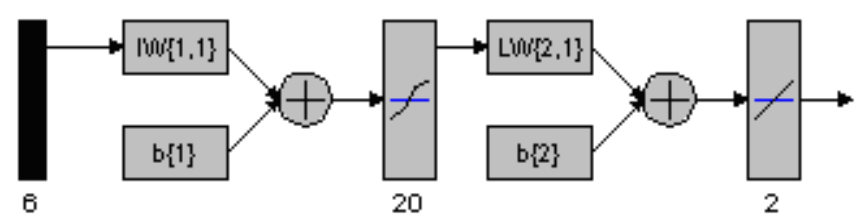

الشكل رقم 3 : المخطط الكتلي للثبكة العصبية الاصطناعية المصمم. 


\section{د - كمية المياه الواردة في السنة التالية :}

تؤثر كمية المياه المتوقعة للسنة التي تلي سنة الدراسة في كمية المياه المخزونة (وبالثالي كمية المياه المطلقة) وذلكك لمعالجة أي خلل في المياه الواردة لتلأك السنة. لذا يجب الحصول على تلى توقعات صديحة لكمية المياه السنوية الو اردة. ثم اعتماد معطيات السنة اللاحقة ضمن المعلومات المثوفرة لفترة الدراسة، ولكن بصورة عامة يتم إيجاد كمية المياه المتوقعة ضمن إدارة الموارد المائية. إن معرفة كمية المياه وكذلك توقع كمية الأمطار بمثل أهمبة خاصة ضمن إدارة الموارد المائية ولدرء الحوادث نتبجة للفيضانات وغيرها.

$$
\text { و هي تحسب في دول متعددة مثل روسبا و أمربكا وكندا وألمانيا وغير ها. }
$$

تحوي التوقعات نسبة من الخطأ نختلف بين طريقة حساب وأخرى وكذلك مدى ملائمة الطريقة لحالة معينة. لذا فان عدم الدقة تؤخذ بنظر الاعثبار ولكن في الجزء الثاني من البحث والمتعلق بالتوزيع لفترة قصبرة (بحث درسل للنشر) .

هـ - كمية المياه المخزونة:

تمثل هذه القيمة كمية المياه المخزونة في بحيرة السد عند بدء الشهر ـ وتبدأ السنة بكمية مياه محالة مسـن

السنة السابقة تلاثم كمية المياه المتوفعة لنسنة الدر استة هذه القيمة هي إحدى المحددات التي يجب ملاحظتها لعدم تجاوز

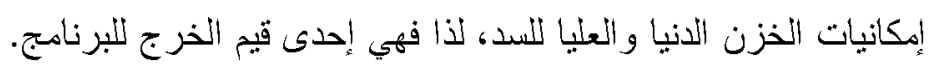

\section{و - كثفة الأحمال الكهربائية الثهرية:}

يختلف الحمل الكهربائي باختلاف أُشهر السنة وذلك بسبب تأثر الظروف المناخية. وتسجل أعلى الأحمال صيفا وشتاءا. تكون اقل الأحمال في فصلي الزبيع والخريف. كذلل تتنوع مصادر الطاقة الكهربائية هن مصادرها

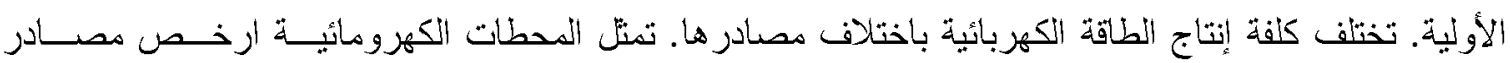

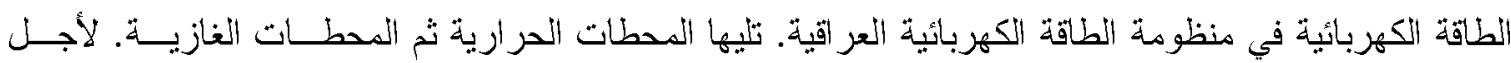
إحلال الطاقة الكهروماثية بديلا عن أُعلى المصادر كلفة، اخذ هذا العامل بنظر الاعتبار .

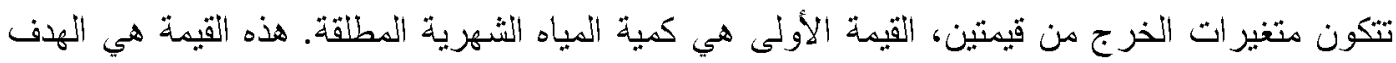

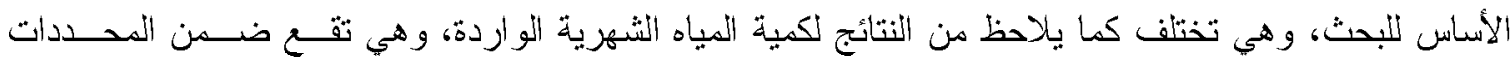

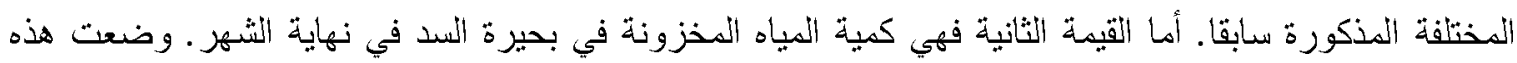
القيمة للتأكد من كون المياه المخزونة في بحيرة السد تقع ضدن الحدود المسموح بها للخزن في بحيرة السد.

تختلف كمية المياه الو اردة في سنو ات منعاقبة كما تختلف كمية المياه الو اردة في أثنهر سنة معينة. يوضح

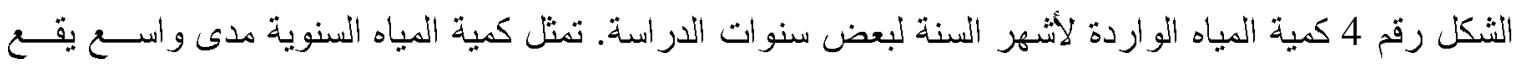

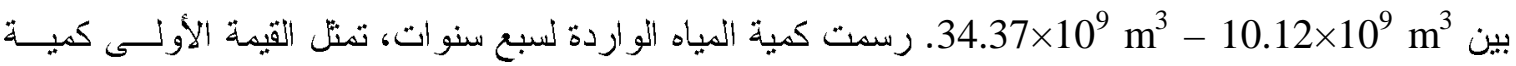

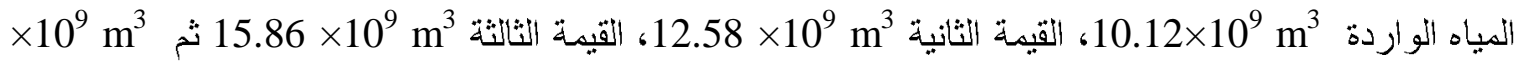

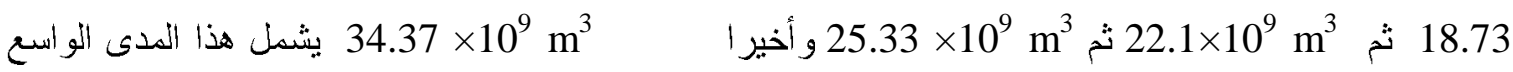
مدى التتبذب السنوي للمياه الو اردة لمدة 65 سنة، تمتن بين سنتي 1931 - 1995. اخذ هذا المدى ليشمل البحــث 
ألحافظ: استخام الثبكة العصبية الاصطناعية في التثغيل الأمثل لمحطات التوليد الكهرومائية للفترة الطويلة

الددى الحقيقي لتتذبذب المياه في نهر دجلة، ليتم الوصول إلى حل حقيقي لهذه المشكلة. استخدت هــــ البيانـات لتكون البيانات المستخدمة لتدريب الثنبكة العصبية الاصطناعية.

\section{كمية المياه الواردة شهريا لسنوات مختارة}
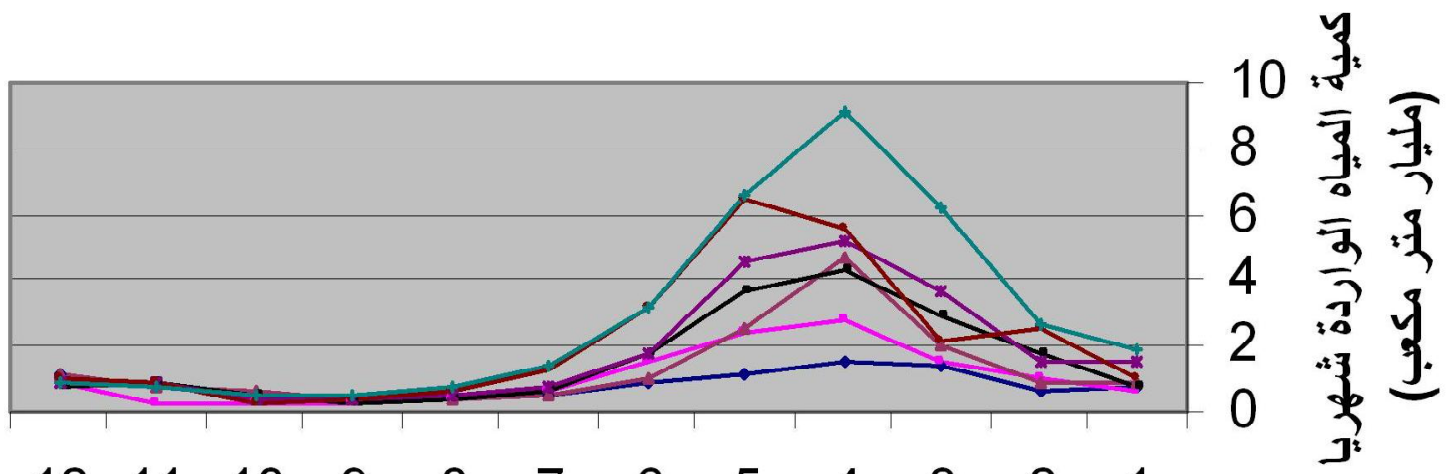

$\begin{array}{llllllllllll}12 & 11 & 10 & 9 & 8 & 7 & 6 & 5 & 4 & 3 & 2 & 1\end{array}$

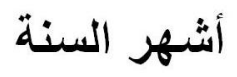

شكل رقم 4 العلاقةّة بين كمية المياه الواردة شهريا لسنوات مختارة.

تحسب الكمية المثالية التي ثقع ضمن المددات وتحقق الهدف بثقليل كلفة توليد الكهرباء وبالاســفادة مــن

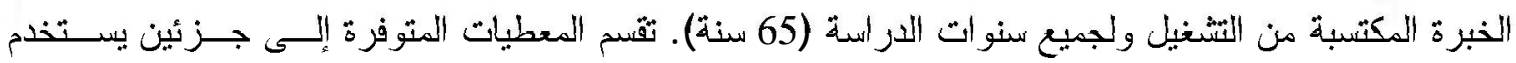

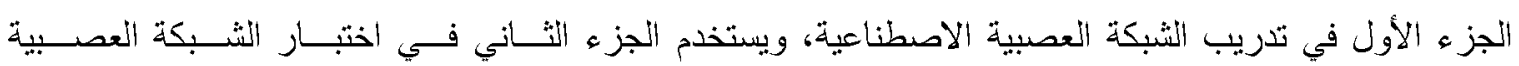

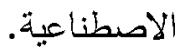

\section{3 - تدريب الثبكة العصبية الاصطناعية :}

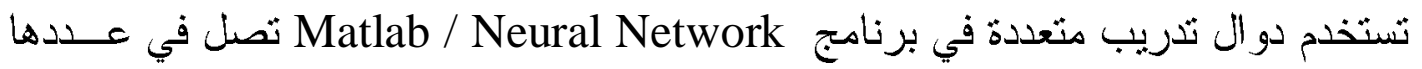

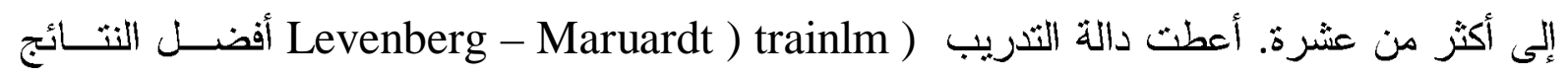
عند تطيقها، لذا اعتمدت للاستخدام في تدريب الثبكة المكونة.

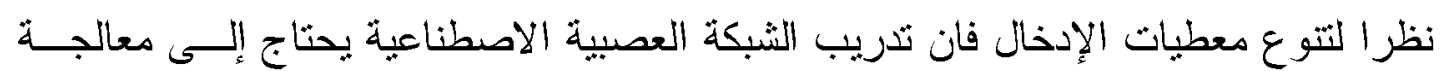

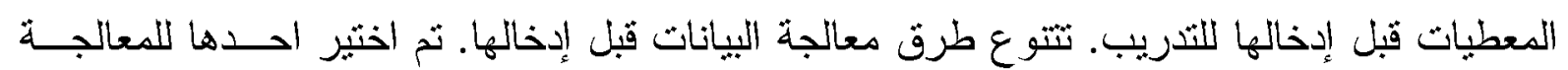

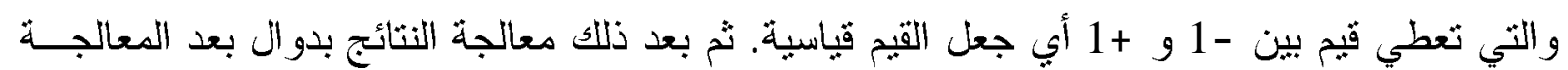

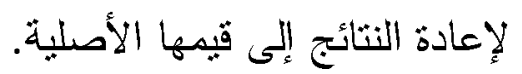

استخدمت المعطيات في فقرة إعداد البيانات و الموضحة في الفقرة السابقة. تغطي البيانات المستخدمة فـي

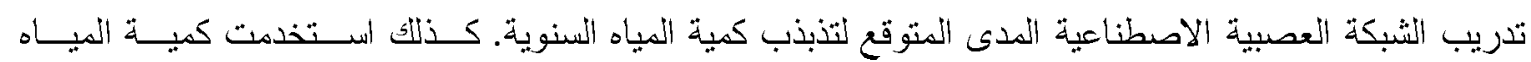

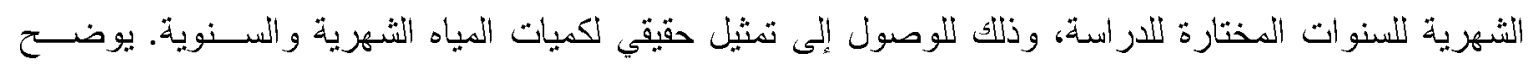




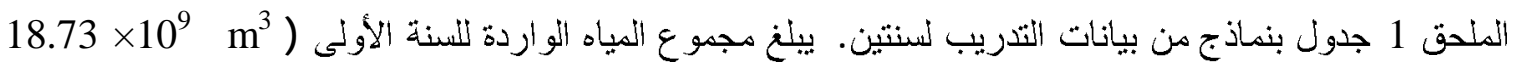

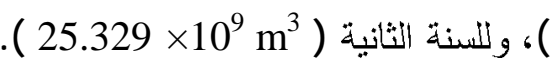

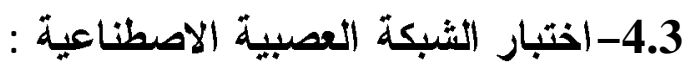

استخدمت معطيات ضمن المدى المتوقع لتنبذب كمية المياه الثنهرية و السنوية (وهو مدى تدريب النشبكة

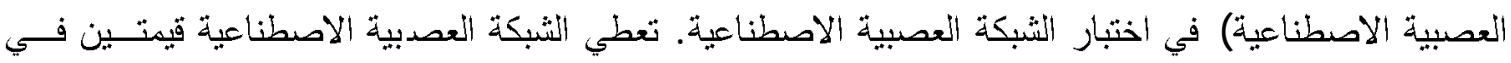
معطيات الإخر اج، هما كمية المياه المطلقة شهريا وكمية المياه المخزونة في نهاية كل شهر .

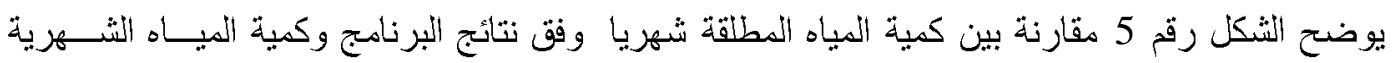

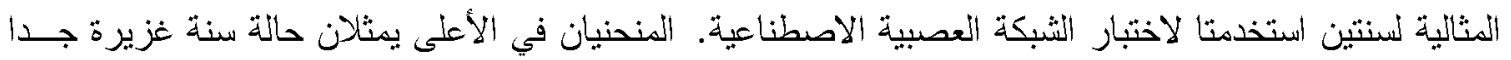

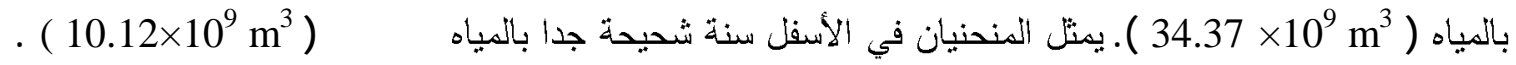

\section{مقارنة للمياه المطلقة شهريا بين الحالة المثالية ونتائج} البرنامج
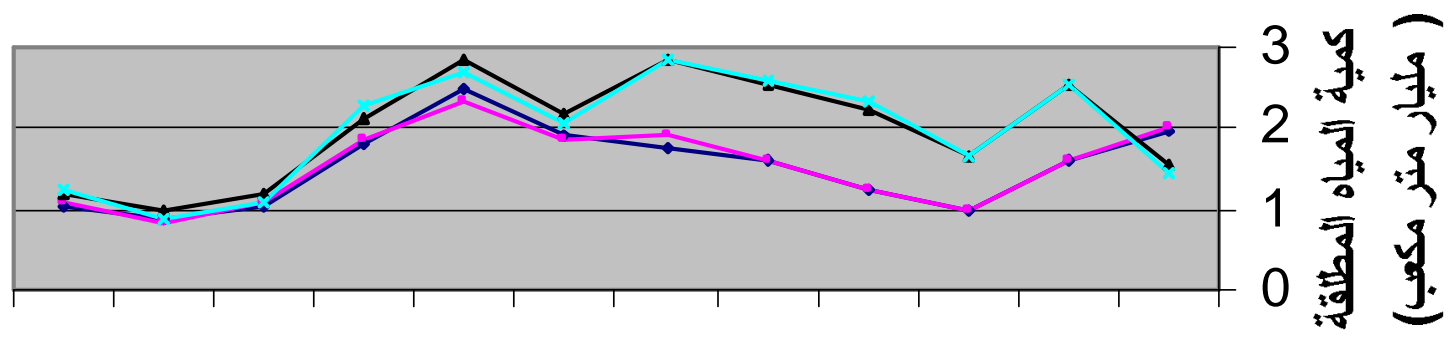

$\begin{array}{llllllllllll}12 & 11 & 10 & 9 & 8 & 7 & 6 & 5 & 4 & 3 & 2 & 1\end{array}$ أشهر السنة

الثكل رقم 5 مقارنة بين المياه المطلقة شهريا للحالة المطلوبة مع نتائج البرنامج.

يوضح الثكل رقم 6 مقارنة بين كمية المياه المخزونة في بحيرة السد لأثتـهر نفـس السـنتين السـابقتين

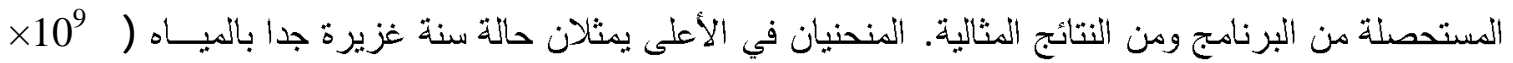

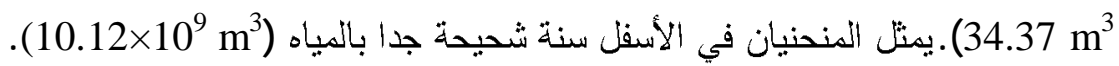

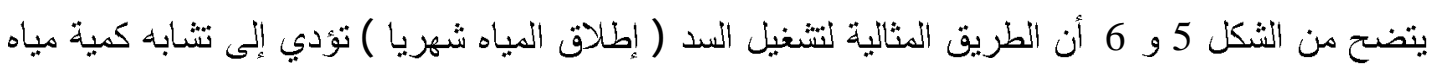

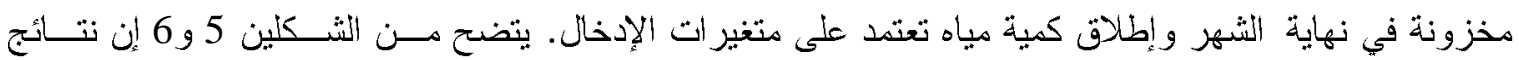

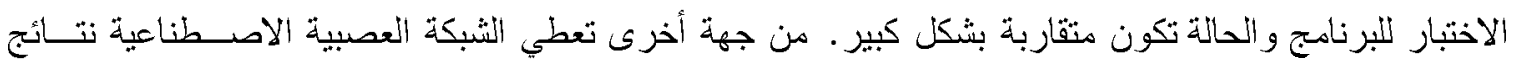
دقيقة 100 \% عند اختبار ها بمعطيات استخدمت في تدريبها. 


\section{4}

يؤدي تذبذب كمية المياه النشهرية و ألسنوية التى عدم الإيفاء بالمتطلبات الثنهرية و الســنوية للاحتياجـات

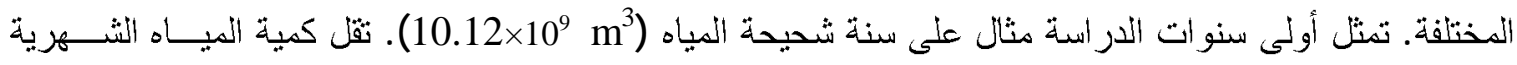

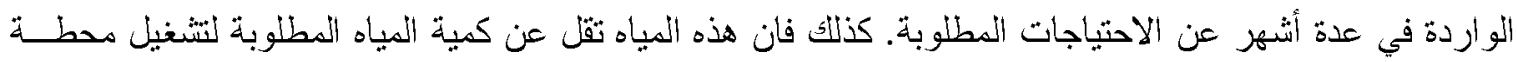

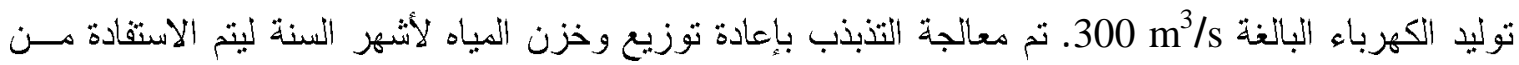

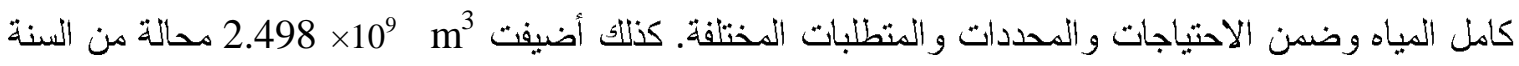

\section{مقارنة المياه المخزونة للحالة المثالية ونتاتج البرنـامج}
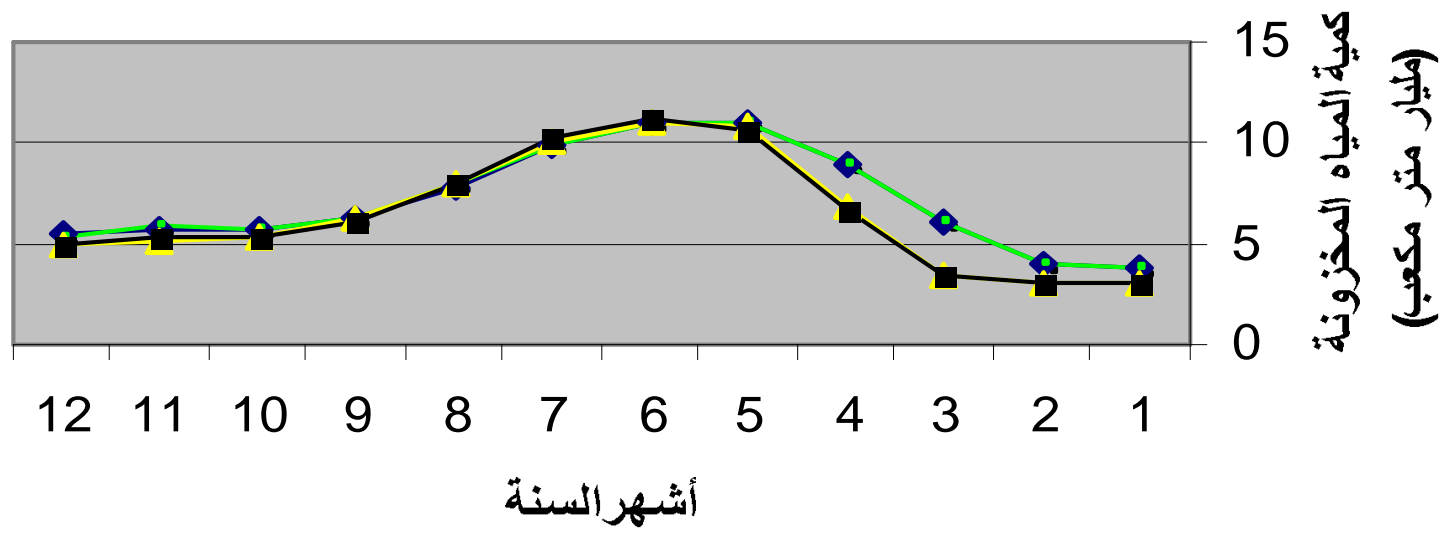

$\begin{array}{llllllllllll}12 & 11 & 10 & 9 & 8 & 7 & 6 & 5 & 4 & 3 & 2 & 1\end{array}$

\section{أشهر (لسنة}

\section{الشكل رقم 6 : مقارنة بين المياه المخزونة شهريا للحالة المطلوبة مع نتائج البرنامج.}

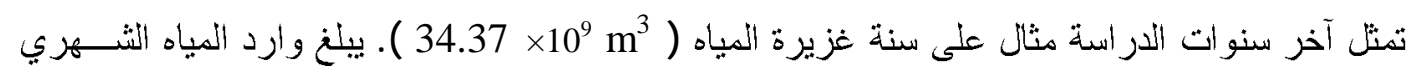

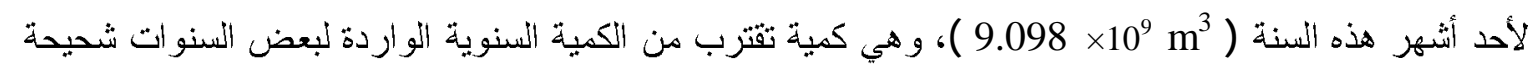

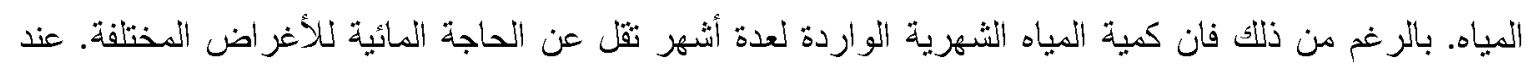

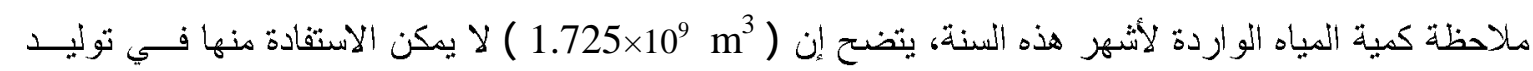

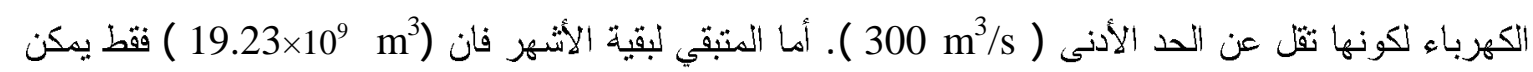

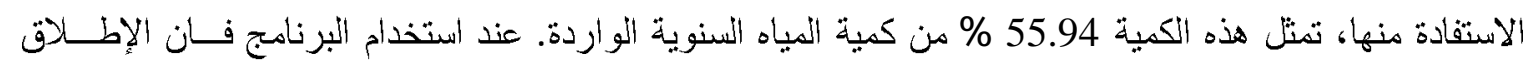
لجميع أُشهر النسنة يقع ضمن الحدود و المتطنبات المختلفة كذلت فان كمية (2.05×109

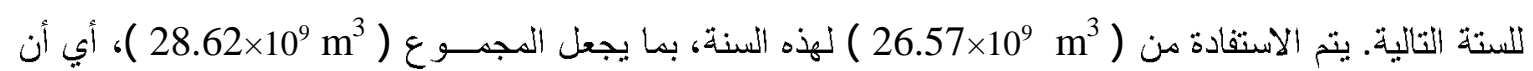
نسبة 83.28 \% من المياه النواردة يتم الاستفادة منها.

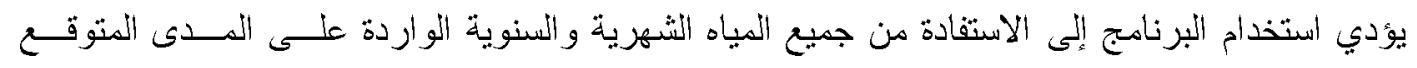
لتنبذب المياه الؤ اردة للشئهر و السنوات، عدا بعض الاستثاءات البسيطة، وضمن المحددات و المتطلبات المختلفة. يتم ذلك بتوزيع الخزن و الإطلاق على أثنهر السنة باستخدام طريقة الثبكة العصبية الاصطناعية. 
إن تذبذب المياه الثهرية والسنوية الو اردة هي إحدى المشاكل الكبيرة التي تو اجه الفنيين و المختصين عند

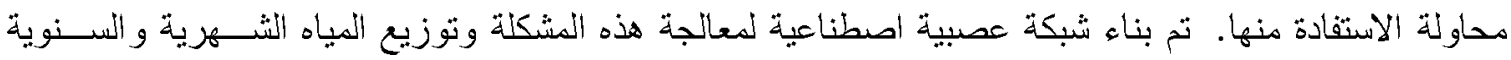

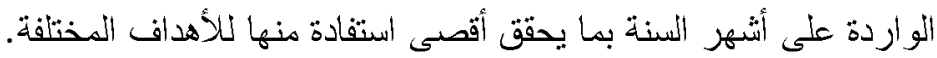

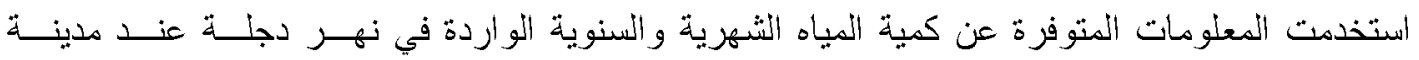

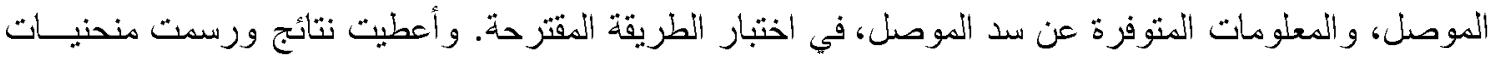
هتعددة لإثبات و إظهار إمكانيات الطريقة المقترحة.

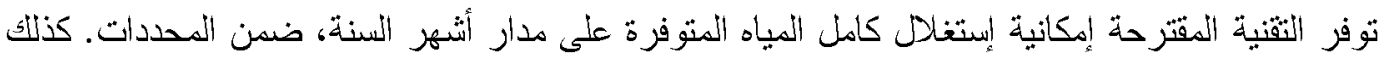

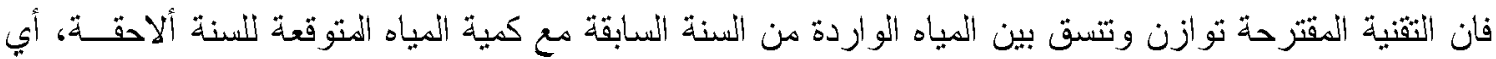

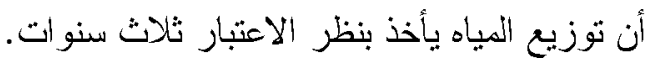

\section{ملحق رقم 1 نماذج من قيم الإدخال}

\begin{tabular}{|c|c|c|c|c|c|}
\hline كلفة الطاقة & المياد المخزونة & المياه الواردة السنة & تسلسل الثهر & الاحتياجات & الثياه الشهرية \\
\hline 297 & 3.834 & 15.8 & 1 & 0.67 & 0.819 \\
\hline 242 & 4.028 & 15.8 & 2 & 0.726 & 1.79 \\
\hline 159 & 5.991 & 15.8 & 3 & 0.937 & 2.946 \\
\hline 201 & 9.000 & 15.8 & 4 & 1.037 & 4.251 \\
\hline 242 & 11.100 & 15.8 & 5 & 1.071 & 3.696 \\
\hline 344 & 11.100 & 15.8 & 6 & 1.166 & 1.778 \\
\hline 446 & 9.898 & 15.8 & 7 & 1.34 & 0.691 \\
\hline 580 & 7.784 & 15.8 & 8 & 1.61 & 0.348 \\
\hline 427 & 6.275 & 15.8 & 9 & 1.166 & 0.303 \\
\hline 239 & 5.724 & 15.8 & 10 & 1.071 & 0.463 \\
\hline 201 & 5.732 & 15.8 & 11 & 0.777 & 0.863 \\
\hline 239 & 5.500 & 15.8 & 12 & 0.67 & 0.782 \\
\hline 297 & 2.95 & 18.7 & 1 & 0.67 & 1.018 \\
\hline 242 & 2.95 & 18.7 & 2 & 0.726 & 2.531 \\
\hline 159 & 3.475 & 18.7 & 3 & 0.937 & 2.201 \\
\hline 201 & 6.803 & 18.7 & 4 & 1.037 & 5.547 \\
\hline 242 & 10.789 & 18.7 & 5 & 1.071 & 6.508 \\
\hline 344 & 11.100 & 18.7 & 6 & 1.166 & 3.162 \\
\hline 446 & 10.136 & 18.7 & 7 & 1.34 & 1.224 \\
\hline 580 & 7.898 & 18.7 & 8 & 1.61 & 0.608 \\
\hline 427 & 6.215 & 18.7 & 9 & 1.166 & 0.412 \\
\hline 239 & 5.304 & 18.7 & 10 & 1.071 & 0.262 \\
\hline 201 & 5.177 & 18.7 & 11 & 0.777 & 0.860 \\
\hline 239 & 5.000 & 18.7 & 12 & 0.67 & 0.996 \\
\hline & & & & & 25.329 \\
\hline
\end{tabular}




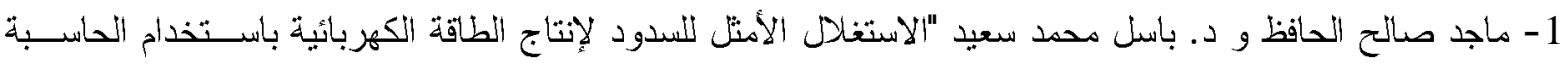

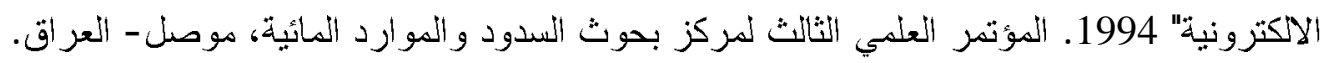

2- H. Dura, C. Pench, and J. Diaz "Optimal operation of multi reservoir system using an aggregation decomposition approach", 1985. IEEE Trans., Vol. PAS-104, PP 2086-2092.

3- V. R. Sherkat, R. Campo, and K. Moslehisk, 1985. "Stochastic long term hydrothermal operation for multi reservoir system" IEEE Trans., Vol. PAS104 ,.

4- E. Gil, J. Buston and H. Rudnick "Short term hydrothermal generation scheduling model using a genetic algorithm", 2003. IEEE Trans. On Power system, Vol.18, No. 4, PP 1256-1264.

5- C. E. Zoumas, A. G. Bakirtzis, J. B. Theocharis and V. Petridis, 2004. "A genetic algorithm solution approach to the hydrothermal coordination problem" IEEE Trans. On Power system, Vol.19, No. 2, PP 1356-1364.

6- Ministry of Irrigation "Discharges for selected gauging stations in Iraq" Baghdad, Iraq.

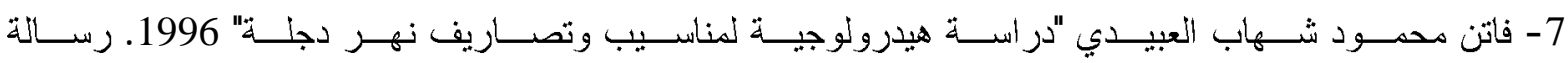

$$
\text { ماجستير ، قسم هندسة المو ارد الماثية، جامعة الموصل. }
$$

8- Warwick, A. Ekwue and R. Aggarwal, 1997. "Artificial intelligence techniques in power systems” IEE Power Engineering Series 22, London.

9- The Math Work Inc., Neural Network Toolbox use with Matlab, 2002, MA, USA

$$
\text { تم اجراء البحث في كلية الهندسة - جامعة الموصل }
$$

